\title{
EVALUATION OF TRANSVERSE DENTAL CHANGES AFTER SURGICAL ASSISTED RAPID MAXILLARY EXPANSION IN ADULTS
}

\author{
Arafa Gad Allah Ibrahim*, Mansour Mohammad Hussein**, Mohammad Abd Elhamied Shuman***
}

\begin{abstract}
Objectives: Surgical assisted rapid maxillary expansion (SARM can affect both skeletal and dental shape and soft tissue the size of the nasal passages and airway resistance, favoring improved nasal respiration. Aim: The aim of this study was to evaluation of transverse dental changes after surgical assisted rapid maxillary expansion in adults. Patients and Methods: The present study was carried out to clinically assessment of the surgical assisted rapid maxillary expansion. Nine healthy adult patients were used in this study. Surgical assisted rapid maxillary expansion was done. Patients were subjected to evaluation by study models. Results: Results of the current study have indicated that surgical assisted rapid maxillary expansion values of study models were higher. Conclusion: study models may be effective in clinically assessment of surgical assisted rapid maxillary expansion.
\end{abstract}

Keywords: surgically assisted rapid maxillary expansion, transverse maxillary dimension

\section{INTRODUCTION}

Transverse expansion of the maxilla was first done in 1860 by means of an orthodontic appliance. Once skeletal maturity has been reached, orthodontic treatment alone cannot provide a stable widening of the constricted maxilla in cases of deficiencies of more than $5 \mathrm{~mm}$. In general, an orthodontist can camouflage transverse discrepancies less than $5 \mathrm{~mm}$ with orthopedic forces alone. Tooth extractions for alignment of dental arches are often unnecessary. Surgically assisted rapid maxillary expansion (SARME) as recommended by Bell and Epker. is a well-established method for correction of transverse maxillary deficits in nongrowing patients. Crossbite and crowded teeth are a typical characteristic of maxillary compression syndromes. ${ }^{1}$ Studies have reported various amounts of dental and skeletal expansion that occur with RME, but it is postulated that RME produces expansion that is $50 \%$ dental and $50 \%$ skeletal. After cessation of growth sutural closure and transverse growth has been completed, orthopedic transverse maxillary expansion is largely unsuccessful because the expansion is primarily composed of alveolar or buccal tipping of posterior maxillary teeth with little or no basal skeletal movement $^{(2,3)}$.

RME and SARME can affect both skeletal and dental shape and soft tissue the size of the nasal passages and airway resistance, favoring improved nasal respiration. Surgically-assisted maxillary expansion can be considered as part of the overall treatment plan for nongrowing patient with a constricted maxillary $\operatorname{arch}^{(4-6)}$.

A surgically assisted rapid maxillary expansion procedure is essentially a combination of osteogenic distraction with controlled expansion of soft tissues. SARME is a form of distraction osteogenesis (DO). In the purest sense, craniofacial DO was first reported in the early 1860's by Angell (7). Advantages of the SARME include improved periodontal health;

\footnotetext{
* Assistant Lecturer of Oral and Maxillofacial Surgery, Faculty of Dental Medicine, (Asiut Branch) Al-Azhar University

** Professor of Oral and Maxillofacial Surgery, Faculty of Dental Medicine (Cairo) Al-Azhar University

*** Assistant professor of Oral and Maxillofacial Surgery, Faculty of Dental Medicine, (Asiut Branch) Al-Azhar University
} 
improved nasal airflow elimination of the negative space, which results in less visible tooth and gum structure showing during smiling; a cosmetic improvement of the buccal hollowing second to post-expansion prominence at the site of the lateral wall osteotomy; and bone apposition in the osteotomy site and reduced risk of dental version or extrusion compared with regular orthopedic care. Also, tooth extraction for alignment of the arches is often unnecessary ${ }^{(8)}$.

\section{PATIENTS AND METHODS}

Patient Selection: Nine Patients were included in the present study. They were consecutive patients selected from the oral and maxillofacial surgery department that referred from orthodontic department Faculty of Dental Medicine, Al-Azhar University, Assuit branch. They were adult patients of both sexes. All patients had maxillary transverse deficiency associated primarily with functional impairments, such as posterior uni- or bilateral cross bite, dental crowding, reduced nasal respiratory function or anterior-posterior skeletal anomalies and they were indicated for surgically assisted rapid maxillary expansion.

Preoperative evaluation, preparation and measurements: History was taken then all patients underwent complete physical examination. Clinical evaluation and examination by inspection and palpation of soft tissue. Impression was taken and study model then mounted on articulator. Intracanine and intramolar distances was measured by digital caliper (Fig.1)

Direct measurements on study models were made with a digital sliding caliper. Measurements were taken at two reference points on the canine's and the first molars respectively, according to Moorrees to measure intramaxillary distance anteriorly and posteriorly. CI denotes the distance between the cusp tips of the canines and CII the distance between the most prominent cervical point of the palatal ridge on the canines. MI represents the distance measured between the mesiobuccal cusps tips of the maxillary first molars and MII the distance between the most cervical points of the palatal fissure of the maxillary first molars.

A Hyrax device installation: hyrax expansion appliance was applied before surgery and was inactivated. A rigid expansion appliance is usually cemented to the first premolars and first molars on each side.

Surgical procedure: Patients will be prepared in general routine manner for operation under general anesthesia. Nasoendotracheal intubation will be used in all cases, scrupulous disinfection of oral cavity and extraoral. After submucosal injection of vasoconstrictor intra oral incision will be made in the depth of the maxillary vestibule, $3-5 \mathrm{~mm}$ above the level of attached gingiva in the alveolar mucosa and leaving unattached mucosa on the alveolus to facilitate closure extended from second premolar on one side to second premolar in other side.

The soft tissues are reflected subperiosteally by periosteal elevator from the lateral aspect of the maxilla, with dissection to expose the anterior floor of the nose and piriform aperture area, posteriorly toward the pterygo-maxillary fissure. Osteotomy by using of surgical bur and the level of the lateral maxillary osteotomies is measured to be at least 5 $\mathrm{mm}$ above the apices of the teeth. As the anterior portion of the osteotomies is being performed, periosteal elevator is maintained in the piriform rim, lifting the nasal mucoperiosteum to protect it. The lateral wall osteotomies are extended posteriorly toward the pterygo-maxillary fissure (Fig. 2). On completion of the lateral maxillary osteotomy the osteotome is malleted posteriorly for approximately $30 \mathrm{~mm}$, to section the lateral nasal wall. Similar osteotomies are performed on the opposite side. The nasal spine and septum are now released with osteotome extending at least $30 \mathrm{~mm}$ posteriorly, having first performed an osteotomy below the 
nasal spine, leaving it attached to the septum and ensuring that the septum remains independent of the maxillary segments. Amidpalatal osteotomy is then accomplished by malletting an osteotome posteriorly, parallel to the palatal plane, into the intermaxillary suture and directed to the posterior nasal spine. These procedures ensure that separation of the entire midpalatal suture has occurred. Before the soft tissue incisions are closed, the expansion screw is turned eight to twelve times, so that the maxilla is expanded 2 to $3 \mathrm{~mm}$ this amount of immediate expansion.

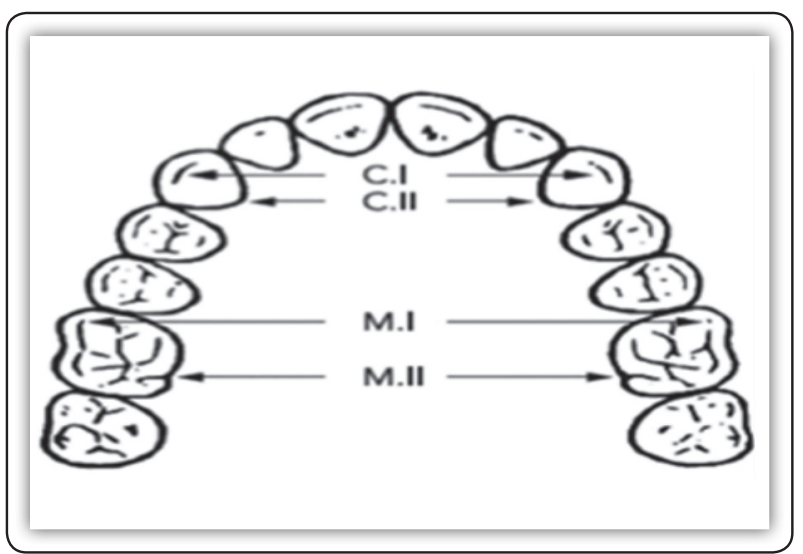

FIG (1) Two reference points on the canines and the maxillary first molars respectively.

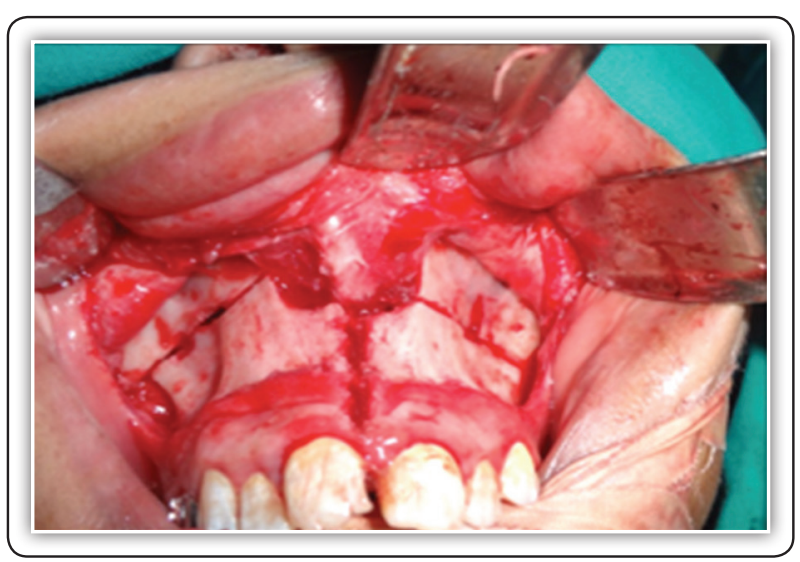

FIG (2) Photograph of bilateral osteotomy.
Expansion protocol: Following the SARME procedure and after seven days' latency period and oral analgesic before expansion for controlling of pain the appliance is activated by the parents of patient, one millimetre each day, until the desired expansion has been achieved. It was expanded to its maximum width, because it is easier to allow the maxillary posterior segments to settle back, if necessary, into ideal transverse relationships with their lower opponents.

Postoperative Evaluation: Clinical evaluations were done at intervals of 2, 4, 7 and 15 days and directed toward the observation of the healing process, infection or any complications of wound healing.

Study model. Intracanine and intramolar distances will be measured by digital caliper after treatment was completed.

\section{RESULTS}

In all patients, the distractor device was well tolerated, remained stable, maintained the osteotomized segments during distraction in the desired alignment. Distraction was carried out manually and continued for 10 days without any difficulty.

Statistical analysis of study model findings. Using a software program SPSS statistical packaged for social sciences V19.0 for windows (SPSS INC, Chicago, USA). Data were presented as mean and standard deviation values. The expansion was statistically significant (Table 1). Depending on the clinical need for expansion, there was a major inter individual variance in the degree of expansion. The greatest expansion was recorded for MI, the distance between the tips of the buccal cusps of the maxillary first molars (Figure 5,6). The mean values of intracanine $(\mathrm{C} 1, \mathrm{C} 2)$ and intramolar distances (M1, M2) from before and after expansion with p-value (0.000) show highly statistically significant differences. 
Table 1. Comparison between maxillary intracanine and intramolar distances for the treated subjects at baseline and after completed treatment

\begin{tabular}{|l|c|c|c|c|}
\hline & Mean (mm) & SD (mm) & t- value & P value \\
\hline \multirow{2}{*}{ C1(B) - C1(A) } & 31.01429 & 0.693851 & \multirow{2}{*}{-26.834} & 0.000 \\
\cline { 2 - 4 } & 34.75714 & 0.450397 & & \\
\hline \multirow{2}{*}{ (C2(B) - C2(A) } & 23.72857 & 0.468025 & \multirow{2}{*}{-6.912} & \multirow{2}{*}{0.000} \\
\cline { 2 - 4 } & 26.58571 & 1.487087 & & \\
\hline \multirow{2}{*}{ M1(B)-M1(A) } & 40.21429 & 1.185227 & & \multirow{2}{*}{0.000} \\
\cline { 2 - 4 } & 46.84286 & 0.962388 & -81.376 & \\
\hline \multirow{2}{*}{ M2(B) -M2(A) } & 25.81429 & 0.654290 & \multirow{2}{*}{-39.025} & 0.000 \\
\cline { 2 - 4 } & 31.71429 & 0.628301 & & \\
\hline
\end{tabular}

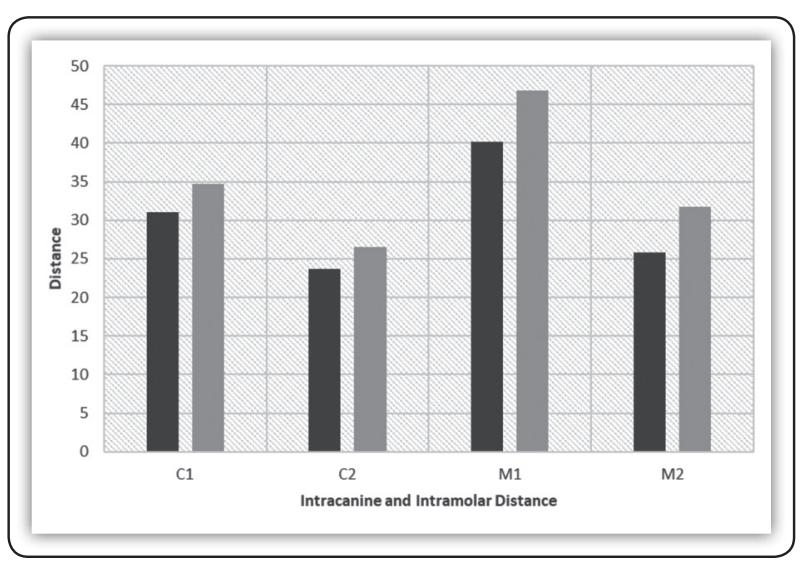

FIG (3) Showing Bar-chart of intracanine and intramolar distances at different intervals of the follow up period.

\section{DISCUSSION}

Various treatment protocols were proposed, all focusing on different aspects of the procedure. The lack of consensus from scientific studies puts clinicians in the challenging position of having to choose and balance between factors such as the complexity of the surgery, risks for the patient, expected long-term stability, the effect on soft tissue, effect on respiration, clinical accessibility for the procedure, etc. The treatment of the patients with this deficient has aimed to transversally increase the diameter of maxilla through orthodontic, orthopedic, and/or surgical procedures. In subjects with skeletal maturity, surgically assisted rapid maxillary expansion has showed good results. surgically assisted rapid maxillary expansion became an overall accepted and common strategy for treatment of maxillary transverse deficiency in non-growing individuals in the late 1980's and early 1990's. ${ }^{(9)}$. Surgically assisted rapid maxillary expansion with attachment of expansion appliances to the buccal teeth and osteotomy of the lateral maxillae and/ or the midpalatal suture has produced satisfactory results in adolescents and adults. Positive secondary effects include widening of the paranasal sinuses, preservation of the palatal arch configuration, and the opportunity to start multibracket therapy for alignment of the maxillary dental arch immediately on completion of the distraction period ${ }^{(10,11)}$. In the current study it is unnecessary to carry out osteotomy in the pterygoid processes. To minimize the surgical trauma, less invasive procedures combined with osteotomies in the bilateral zygomatic maxillary crest and median palatine suture were performed by Kaban and Pogrel et al ${ }^{(12-14)}$. The present study, was to evaluate transverse dental changes after SARME treatment. The dental changes may reflect skeletal displacements, but it is hazardous to draw any major conclusions of skeletal effect on study models alone. There are several accepted indices for evaluating transverse dental deficiencies on study models, such as Pont's Index, Korkhaus Index and Howe's Analysis ${ }^{(15,16)}$. Quimby et al. ${ }^{(17)}$ reported acceptable accuracy but greater variance for measurements made from computer-based models. Thus, the method selected for the present study was direct measurement on study models, using digital calipers. On average, there was greater expansion posteriorly, at the molars, than anteriorly, at the canines. This finding is in agreement with current studies on study models. A number of older studies however, have reported the opposite, greater expansion anteriorly than posteriorly ${ }^{(18,19)}$. 
All posterior crossbites were corrected and the expansion was statistically significant. Depending on the clinical need for expansion, there was a major interindividual variance in the degree of expansion. The greatest expansion was recorded for MI (the distance between the tips of the buccal cusps of the maxillary first molars.

\section{REFERENCES}

1. Bell WH, Epker BN. Surgical orthodontic expansion of the maxilla. Am J Orthod 1976; 70: 517-28.

2. Stevens K, Bressmann T G, Siew-Ging T, Bryan D. "Impact of a rapid palatal expander on speech articulation". Am J Orthod Dentofacial Orthop. 2011; 140: 67-75.

3. Haas AJ, Andrew J, Cuyahoga F. Rapid expansion of the maxillary dental arch and nasal cavity by opening of the midpalatal suture. Angle Orthod 1961; 31:73-90.

4. Basciftci FA, Mutlu N, Karaman AI, Malkoc S, Küçükkolbasi H. Does the timing and method of rapid maxillary expansion have an effect on the changes in nasal dimensions? Angle Orthod 2002; 72: 118-23.

5. Lobato HI, Machado SM, Ribeiro SM. Airway flow and audiologic ability evaluation after rapid maxillary expansion case report. Int J Pediatric Otorhinolaryngology Extra 2010; 5:89-90.

6. Adkins MD, Nanda RS, Currier GF. Arch perimeter changes on rapid palatal expansion. Am J Orthod Dentofac Orthop 1990; 97:194-99.

7. Korn EL, Baumrind S. Transverse development of the human jaws between the ages of 8.5 and 15.5 years, studied longitudinally with use of implants. J Dent Res 1990; 69:1298-306.

8. Bruno R, Chrcanovic 1, Nero C. Orthodontic or surgically assisted rapid maxillary expansion. Oral Maxillofac Surg 2009; 13:123-37.
9. Lanigan DT, Mintz SM. Complications of surgically assisted rapid palatal expansion: review of the literature and report of a case. J Oral Maxillofac Surg 2002; 60:104-10.

10. Bays RA, Greco JM. Surgically assisted rapid palatal expansion: An outpatient technique with long-term stability. J Oral Maxillofac Surg 1992; 50:110-13.

11. Verstraaten, J. A systematic review of the effects of bone borne surgical assisted rapid maxillary expansion. J Cranio Maxillofac Surg 2010; 38, 166-74.

12. Freitas RR, Gonçalves AJ, Moniz NJ, Maciel FA. Surgically assisted maxillary expansion in adults: prospective study. Int J Oral Maxillofac Surg 2008; 37:797-804.

13. Kaban LB. Surgical orthodontic correction of transverse maxillary deficiency: a simplified approach. Plast Reconst Surg 1984; 73:67-68

14. Aukema AA, Fokkens, WJ. Chronic rhinosinusitis: management for optimal outcomes. Treat Resp Med 2004; 3:97-105.

15. Dause R, Cobourne M, McDonald F. Space planning sensitivity and specificity: Royal London Space Planning and Korkhaus Analyses. Aust Orthod J 2010; 26:42-8

16. Joondeph D. R, Riedel R A, Moore A W. Ponts index: a clinical evaluation. Angle Orthod 1970; 40: 112-18.

17. Quimby M L. The accuracy and reliability of measurements made on computer-based digital models, Angle Orthod 2004; 74: 298-303.

18. Wriedt S1. Surgically assisted rapid palatal expansion. An acoustic rhinometric, morphometric and sonographic investigation, J Orofac Orthop 2001; 62:107-15.

19. Sagar D, Chitra S, Srinivas S. Modified SARME (Surgically Assisted Rapid Maxillary Expansion) in Conjunction with Orthodontic Treatment-A Case Report. J Clin Diagn Res 2015; 9:20-22. 